\title{
Measurement and Simulation of the RHIC Abort Kicker Longitudinal Impedance
}

\author{
N.P. Abreu, E. Choi, and H. Hahn
}

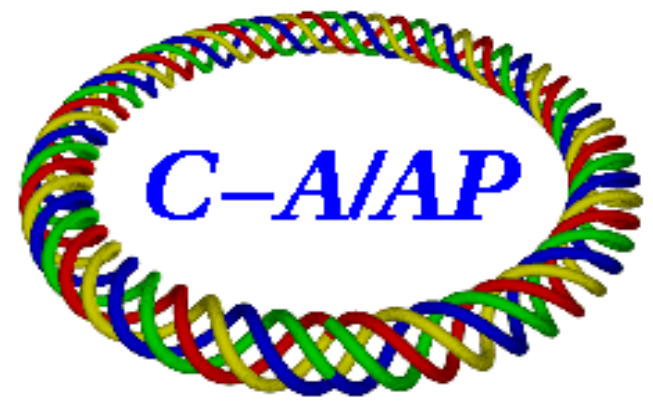

\section{Collider-Accelerator Department Brookhaven National Laboratory \\ Upton, NY 11973}

Notice: This document has been authorized by employees of Brookhaven Science Associates, LLC under Contract No. DE-AC02-98CH10886 with the U.S. Department of Energy. The United States Government retains a nonexclusive, paid-up, irrevocable, world-wide license to publish or reproduce the published form of this document, or allow others to do so, for United States Government purposes. 


\title{
Measurement and Simulation of the RHIC Abort Kicker Longitudinal Impedance
}

\author{
N.P. Abreu, E. Choi and H. Hahn
}

\begin{abstract}
In face of the new upgrades for RHIC the longitudinal impedance of the machine plays an important role in setting the threshold for instabilities and the efficacy of some systems. In this paper we describe the measurement of the longitudinal impedance of the abort kicker for RHIC as well as computer simulations of the structure. The impedance measurement was done by the $\mathrm{S}_{21}$ wire method covering the frequency range from $9 \mathrm{kHz}$ to $2.5 \mathrm{GHz}$. We observed a sharp resonance peak around $10 \mathrm{MHz}$ and a broader peak around $20 \mathrm{MHz}$ in both, the real and imaginary part, of the $\mathrm{Z} / \mathrm{n}$. These two peaks account for a maximum imaginary longitudinal impedance of j15 $\Omega$, a value an order of magnitude larger than the estimated value of $\mathrm{j} 0.2 \Omega$, which indicates that the kicker is one of the main sources of longitudinal impedance in the machine. A computer model was constructed for simulations in the CST MWS program. Results for the magnet input and the also the beam impedance are compared to the measurements. A more detail study of the system properties and possible changes to reduce the coupling impedance are presented.
\end{abstract}

\section{INTRODUCTION}

The RHIC abort kicker is constructed as a window-framed magnet, deflecting the beam horizontally. The kicker is configured of two C-magnets separated by a copper stripe for impedance reduction, as shown in Fig. 1. The magnet is assembled with CMD5005 ferrite pieces, each $20.32 \mathrm{~cm}$ long, for a total length of $122 \mathrm{~cm} \mathrm{[1,2].} \mathrm{The} \mathrm{kicker} \mathrm{magnet} \mathrm{is}$ located in a tubular vacuum vessel and the connection with the Pulse Forming Network (PFN) is through a coaxial feedthrough at a gap in the middle of the side ferrites of the magnet, as show in Figure 2. The abort system in one ring involves 5 equal kickers each separately powered. The measurements reported here were performed on a spare magnet outside of the ring without a PFN connected. 


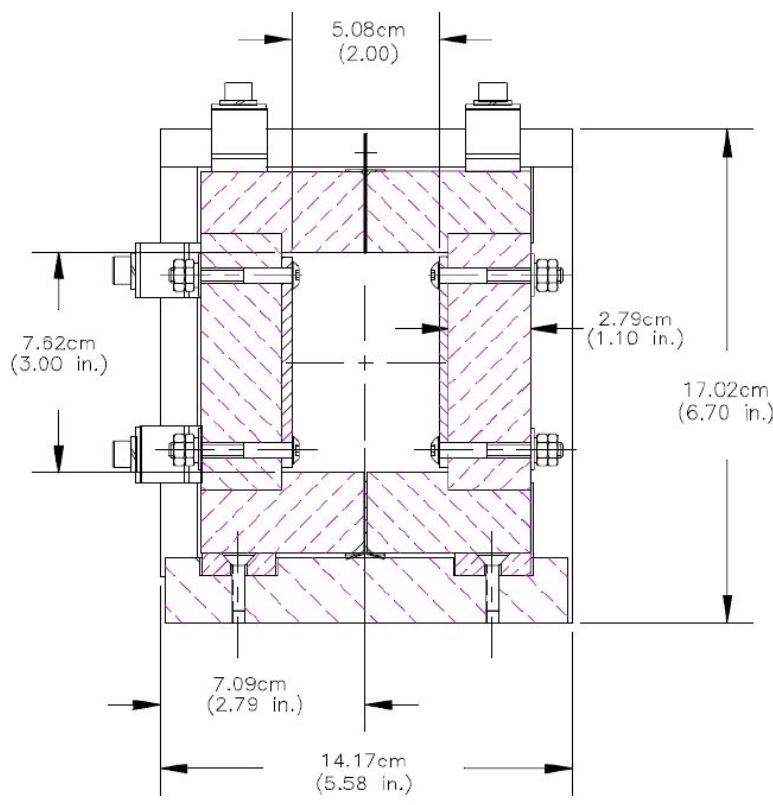

FIGURE 1: RHIC Abort kicker cross section
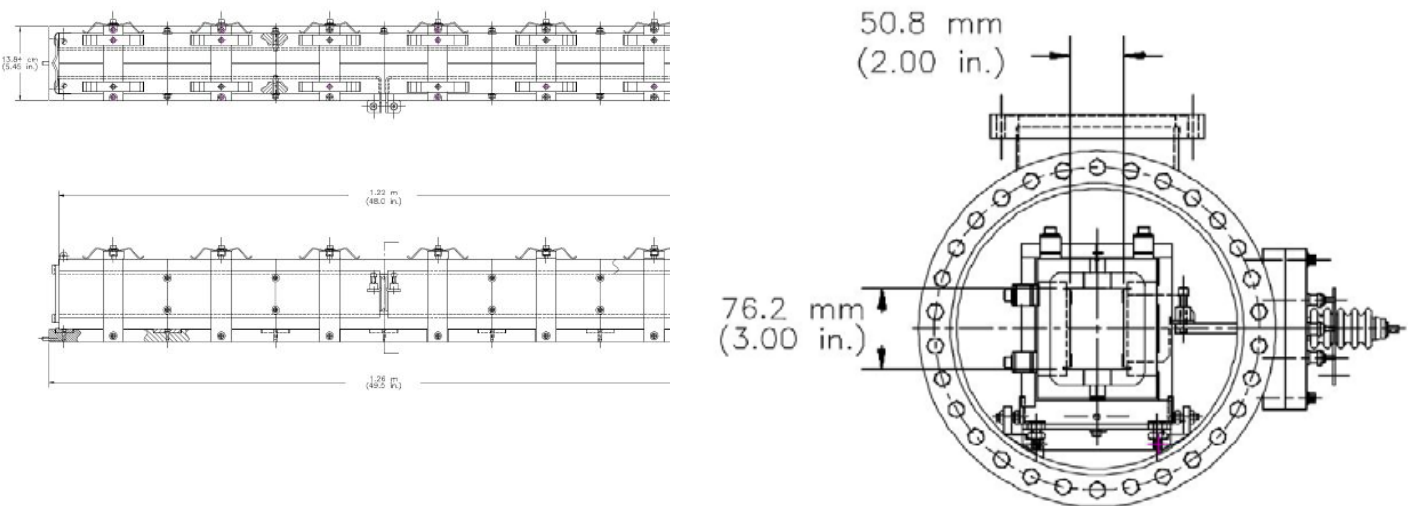

FIGURE 2: End view in vessel and side view of the abort kicker

In this report measurement results for the longitudinal coupling impedance of a single abort kicker are presented, but extrapolation to the impedance of the abort system is left to the reader. The coupling impedance was measured with a network analyzer by the established S21 wire method which, in order to yield accurate results, is best interpreted via the "log-formula" for a distributed impedance [3]. We also measured the impedance at the busbar connections in the kicker side gap either directly or through the feedthrough. The reflected signal (S11) provides a complementary view of the characteristic of the unit and helps in the identification of the resonances. Using CST Microwave Studio computer program we modeled and simulated the impedance of one kicker unit to try to understand the nature of the impedance measured and to get an idea on the properties of the kicker. 


\section{MESUREMENT SETUP}

The coupling impedance was measured using the wire method, in which the beam is represented by a wire and the propagation of a TEM wave in the kicker is compared to that in a coaxial reference tube of equal length. The reference tube must have the diameter of the beam tube which would be replaced by the kicker in the ring. A resistive match provides a smooth transition from the $50 \Omega$ of the Network Analyzer to the characteristic impedance of the reference line in the setup. Using this method it is possible to display the results for the real and imaginary part of the impedance directly on the screen of the network analyzer without any post processing, however for a more accurate calculation we applied the "log-formula" to the S21 data which is more appropriate for distributed systems. Having the S21 reference stored in the network analyzer allowed making repeat measurements on the kicker over a longer period without switching from reference to the DUT.

The longitudinal coupling impedance of the abort kicker (Device Under Test - DUT) was measured with a setup as shown in Figure 3. We used two different wires, a rod with $0.95 \mathrm{~cm}$ and a wire with $0.16 \mathrm{~cm}$ of diameter. The characteristic impedance of the rod and wire in a reference cylindrical beam tube with its $25 \mathrm{~cm}$ diameter is calculated to be $Z_{\text {rod }}=164.8 \Omega$ and $Z_{\text {wire }}=270.4 \Omega$. The input and output resistors necessary to match the $50 \Omega$ of the spectrum analyzer were $[4,5]$ :

- Rod: At the input port a parallel resistor (R1) of $\sim 60 \Omega$ and a series resistor (R2) of $\sim 137 \Omega$ provide forward and backward matching. At the output port a series resistor (R3) of $\sim 115 \Omega$ provides forward matching;

- Wire: At the input port a parallel resistor (R1) of $\sim 55 \Omega$ and a series resistor (R2) of $\sim 244 \Omega$ provide forward and backward matching. At the output port a series resistor (R3) of $\sim 220 \Omega$ provides forward matching.

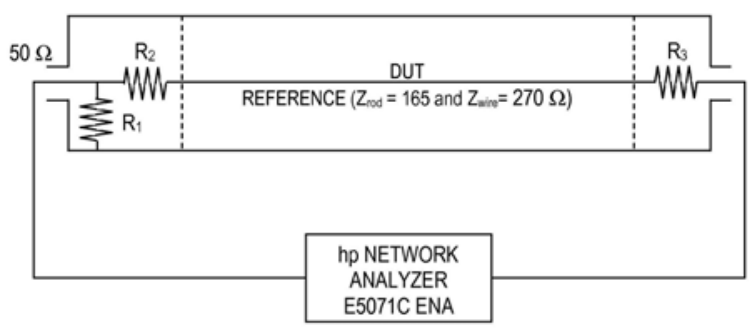

FIGURE 3: Measurement setup

The coupling impedance measurement is obtained from the changes in the forward scattering coefficient $S_{21 D U T}$ in the kicker with respect to the $S_{21 r e f}$ of the reference tube. The general relation between the forward scattering coefficients for a distributed wall impedance is best approximated by the so called "log-formula" [3]

$$
Z(\omega)=2 Z_{0} \ln \frac{S_{21 D U T}}{S_{21 r e f}}
$$


where $Z_{0}$ is the characteristic impedance of the reference, in this case the cylindrical beam tube with wire.

We also connected the Network Analyzer to the busbar connection in the kicker gap either with the feedthrough or directly to the plates for the reflection coefficient measurement. The network analyzer converts the $S_{11}$ directly into an impedance at this port using that $S_{11}=R+j X$ and the following relations:

$$
\begin{aligned}
& \operatorname{Re} Z_{\|}=\frac{1-R^{2}-X^{2}}{(1+R)^{2}+X^{2}} Z_{N A} \\
& \operatorname{Im} Z_{\|}=\frac{-2 X}{(1+R)^{2}+X^{2}} Z_{N A}
\end{aligned}
$$

where $Z_{N A}=50 \Omega$ is the input impedance of the Network Analyzer..

\section{IMPEDANCE MEASUREMENT RESULTS}

We measured the $S_{21}$ parameters for various configurations of the kicker and also measured the $S_{11}$ between the gap plates and the feedthrough. The abort kicker is a lumped inductor and the longitudinal impedance is closely connected to the input impedance at the feedthrough. The PFN was not available for connection to the magnet and its effect on the coupling impedance is expected to be between a $50 \Omega$ termination and a short at the feedthrough.

\section{- Longitudinal Impedance}

We measured two different configurations of the kicker, one with the ends of the feedthrough open and one with its end shorted. The situation in which the feedthrough is attached to the PFN should be an intermediate case between the ones shown. The real and imaginary part of the impedance normalized by the mode number $(\mathrm{Z} / \mathrm{n})$ up to $2.5 \mathrm{GHz}$ is show in Fig. 4. Notice that the kicker does not represent a high impedance source for frequencies above $100 \mathrm{MHz}$, but has a very high peak at low frequencies. With a linear frequency scale, Fig. 5 shows the impedance results only up to $100 \mathrm{MHz}$. In this frequency span it is possible to identify two pronounced peaks in the impedance, one around $10 \mathrm{MHz}$ and the other, much broader, with the maximum between 20-40 MHz. 

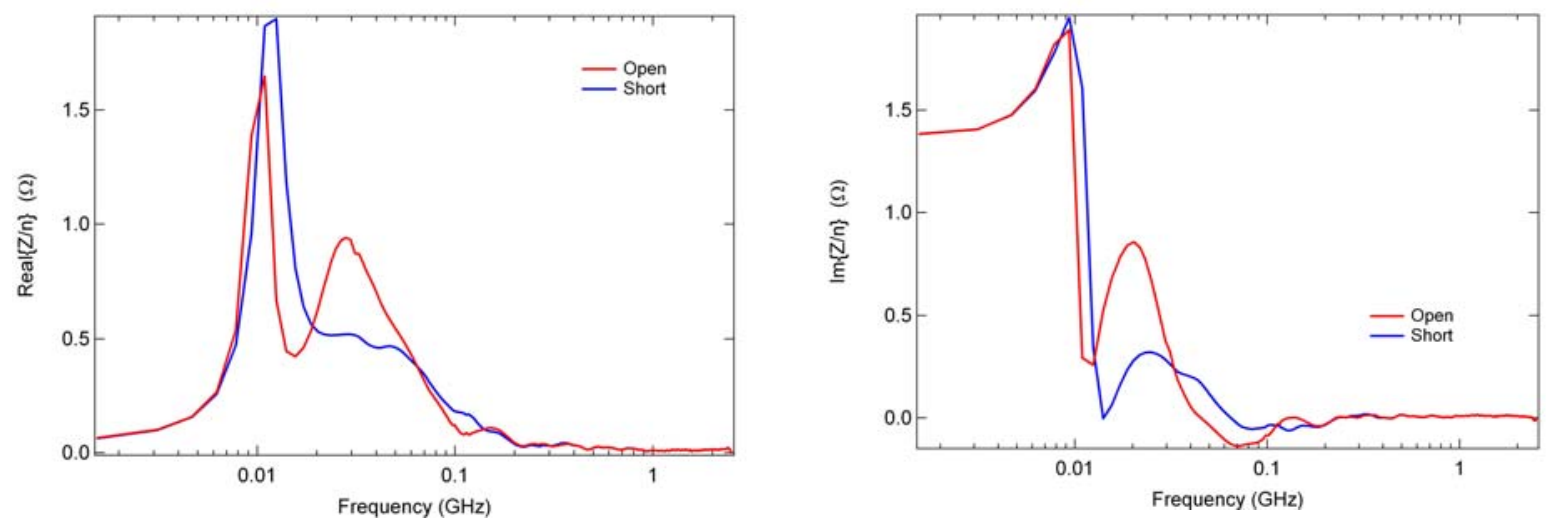

FIGURE 4: Real and Imaginary part of the kicker impedance up to $2.5 \mathrm{GHz}$ with the feedthrough Open and Short.
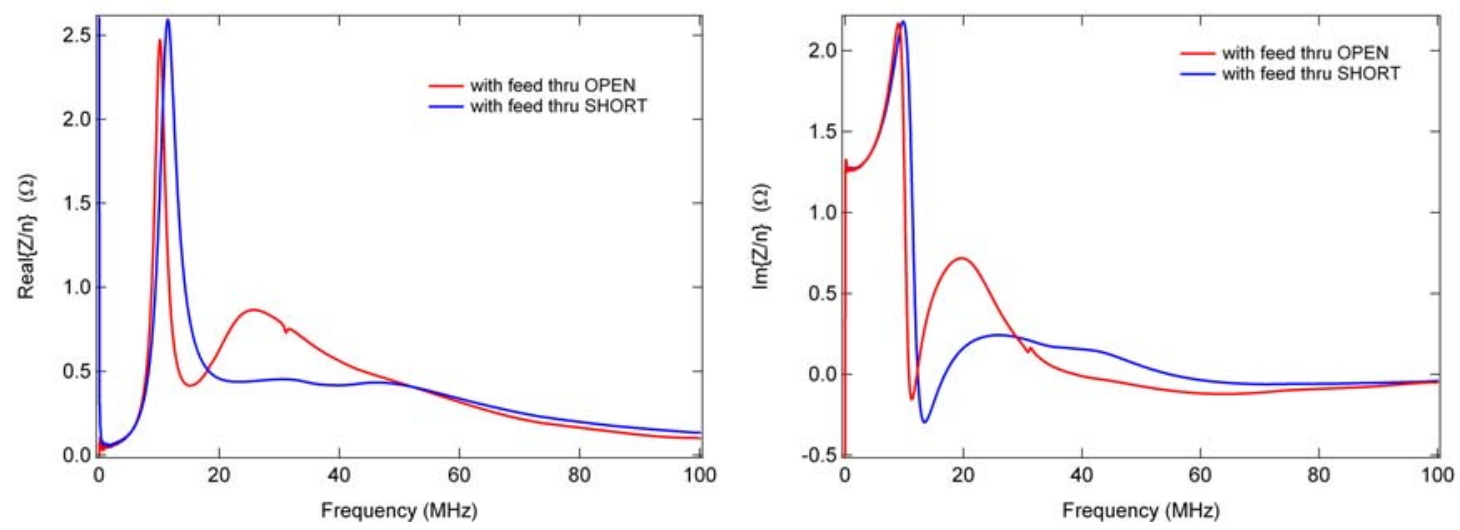

FIGURE 5: Real and Imaginary part of the kicker impedance for the low frequency part of the spectrum with the feedthrough Open and Short.

\section{- Transverse Impedance}

Detailed transverse impedance measurements were previously performed and are shown here in Fig. 6 [ 6].
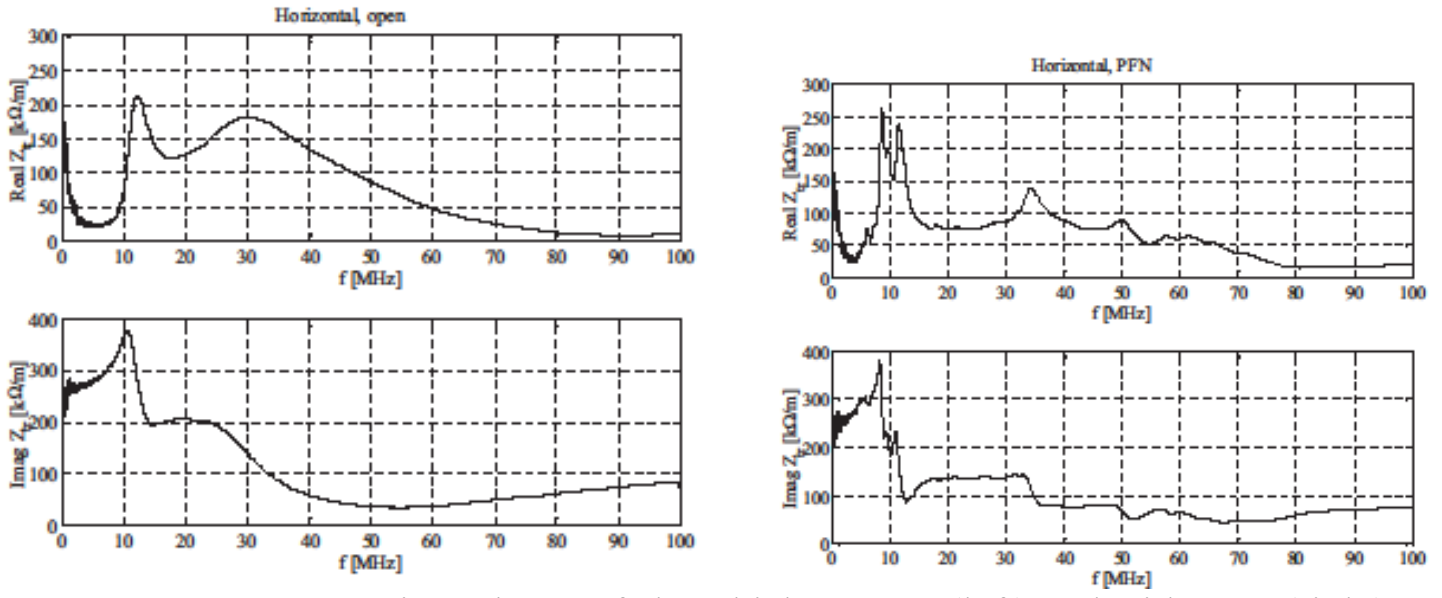

FIGURE 6: Transverse impedance of abort kicker, open (left) and with PFN (right) [6] 
The transverse impedance shows a first resonance at $\sim 10 \mathrm{MHz}$ and a second at $\sim 30 \mathrm{MHz}$ in apparent confluence with the longitudinal impedance results. Terminating the busbar with $25 \Omega$ provided strong damping of the resonances (not shown here). The PanofskyWenzel relation connects transverse and un-normalized longitudinal impedance, according to

$$
Z_{\perp}=\frac{2}{k b^{2}} Z_{\|}
$$

and a comparison can serve as guide to finding a reduction of the coupling impedance. The PFN was not available for the present measurements and in order to try reducing the longitudinal impedance, especially for the broader peak, we attached a $50 \Omega$ resistor to the feedthrough, as show in Fig. 7. The impedance with the load is much closer to the case with the shorted feedthrough. The PFN load represents only a few ohms and connecting it would bring the impedance even more to the shorted case. However, according to instability calculations (private communication M. Blackiewicz) it does not reduce the synchrotron tune shift created by this broad band impedance.
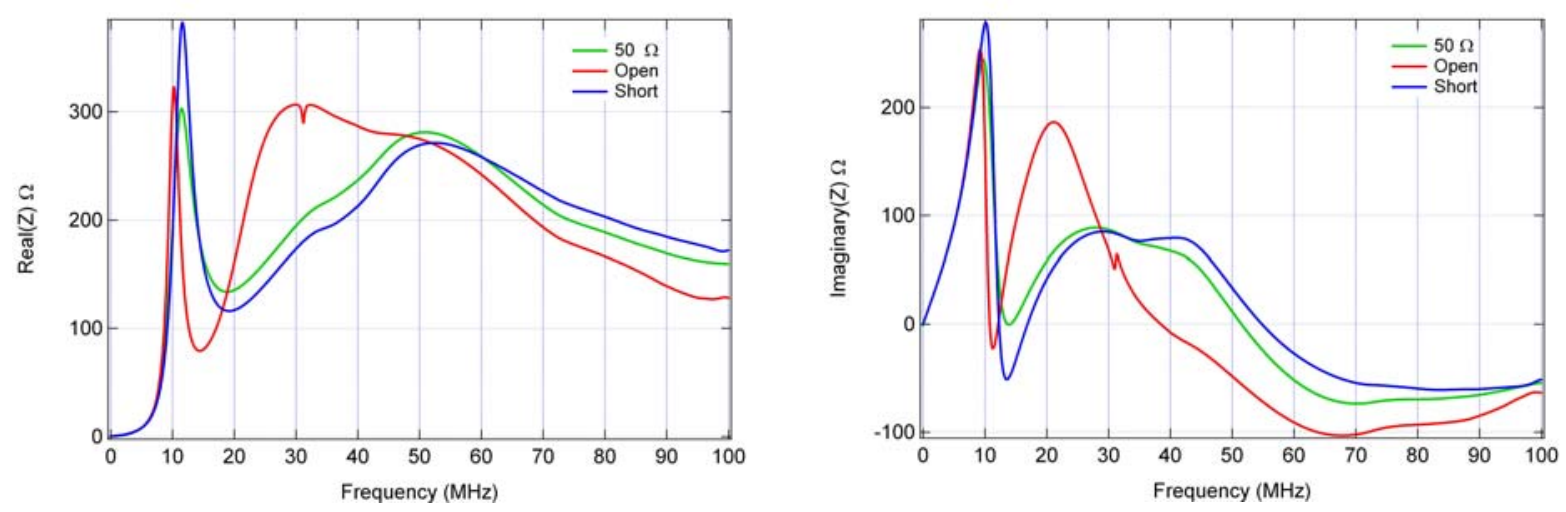

FIGURE 7: Real and Imaginary part of the kicker impedance with the feedthrough Open, Short or a $50 \Omega$ load.

\section{- Busbar Impedance}

Identifying the character and origin of the two resonances is advanced by measuring the input impedance of the busbar at the ferrite gap, shown in Fig. 8. It is noted that the relative amplitude of resonances is changed. Assuming tentatively that the resonances are either longitudinal or transverse, and since by design the busbar couples primarily to the transverse, it is a good conjecture that the $\sim 30 \mathrm{MHz}$ resonance is transverse. The small $80 \mathrm{MHz}$ resonance depends on the feedthrough capacity of $\sim 112 \mathrm{pF}$ interacting with the $\sim 1 \mu \mathrm{H}$ kicker inductance. 

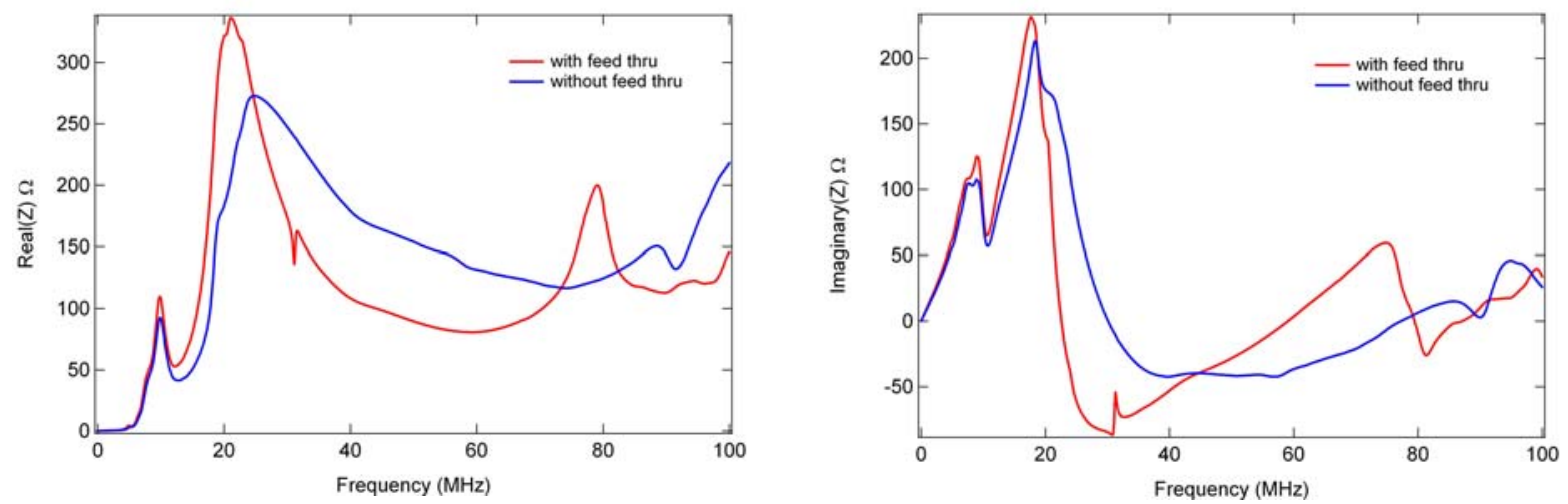

FIGURE 8: The impedance seen at the busbar terminals

\section{KICKER SIMULATION}

Using the program CST Microwave Studio we develop a model for the kicker. In this model the kicker if formed by a window-frame ferrite block. Between the left and right $\mathrm{C}$ shaped ferrite block there is a metallic stripe representing the "eddy current" shield, which is used to reduce the longitudinal coupling impedance of the device. The busbar is inside the kicker window, placed on the lateral wall as a metallic plate from one end to the other, bending only at the gap in the middle and at the kickers ends, as shown in Figure 9. In the simulation for the current we used a metallic rod and two cylindrical metallic terminations. The kicker magnet was constructed with the standard CMD5005 ferrite for which the properties are taken as [7]

$$
\mu_{r}(\omega)=\mu^{\prime}-j \omega \mu^{\prime \prime}=\frac{\mu_{i}-1}{1+j \omega / \omega_{s}}
$$

with $\mu^{\prime}=1600$ and $\omega_{S} / 2 \pi=2.7 \mathrm{MHz}$.
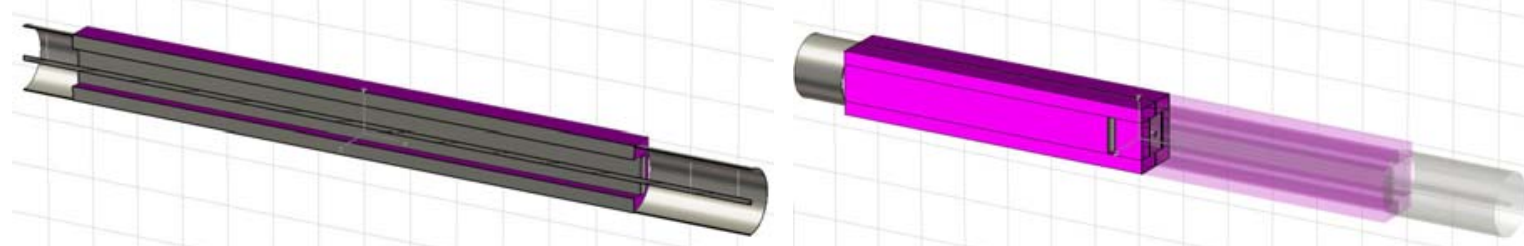

FIGURE 9: Model of the kicker used in the simulations. The magenta structure is the ferrite and the gray parts are perfectly conducting metal

Previous studies for the SNS extraction kicker suggested that using a low-mu ferrite such as C2050 with $\mu^{\prime}=100$ and $\omega_{S} / 2 \pi=55 \mathrm{MHz}$ could lead to a lower coupling impedance [7]. Searching for a method to reduce the coupling impedance, we used the two different types of ferrite, CMD5005 and C2050. The simulation results in Fig. 8 show a sharper resonance for the low-mu ferrite and changes in this direction are not promising. 

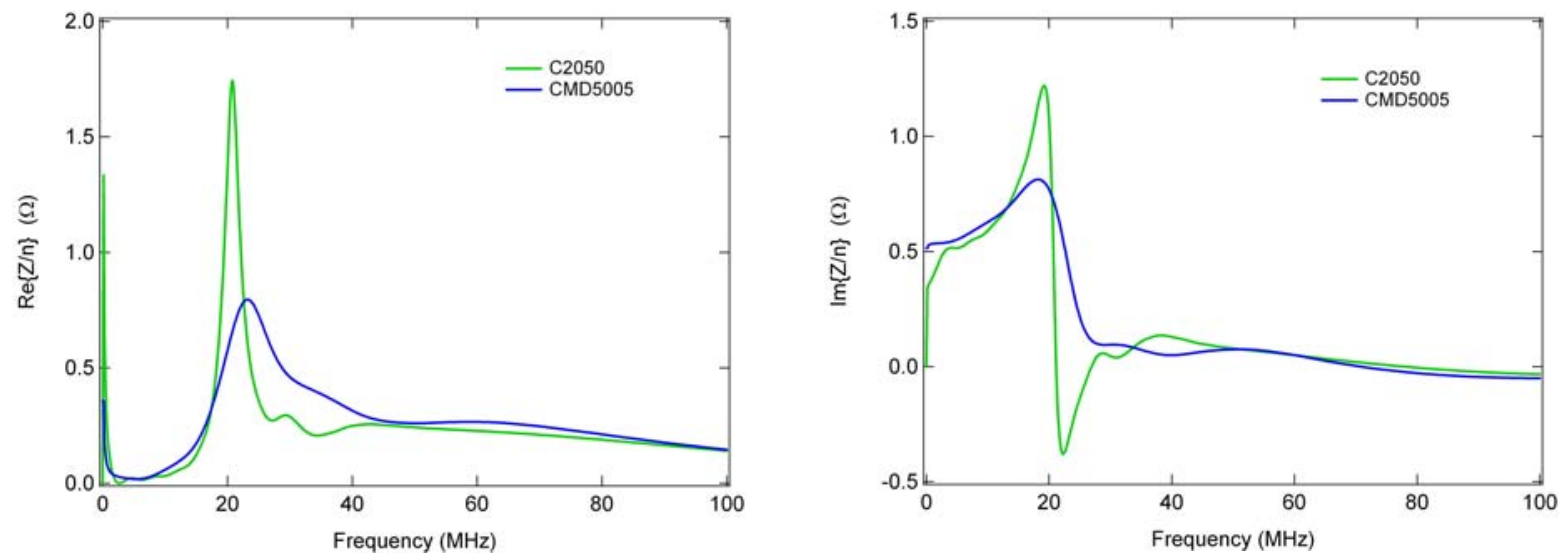

FIGURE 10 : Simulation with two different ferrites

\section{- Resonance identification}

The Microwave Studio simulation produces one clear resonance at a frequency close to the second measured peak. Trying to identify the source of the measured resonance peaks in the CMD5005 magnet, several different values for the ferrite permittivity were used. Very large $\varepsilon$-values did not allow the program to converge but two, $\varepsilon^{\prime}=10$ and 1 , are shown in the plots of Figure 11. The simulation resonance frequency clearly depends on the ferrite parameters according to the expected $\sqrt{\varepsilon}$ dependence but cannot yet be associated with either measured resonance.
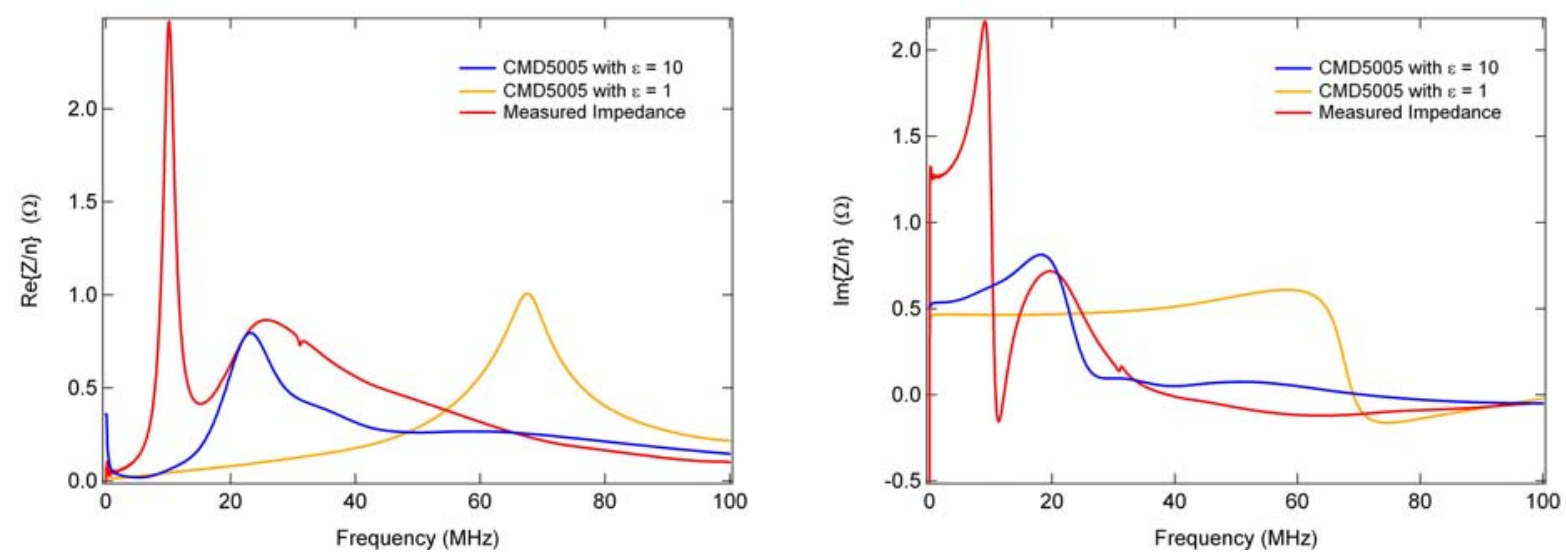

FIGURE 11: Simulation with different permittivity $(\varepsilon)$.

The busbar impedance tentatively suggested the field configurations for the first and second resonance as monopole and dipole. We tested this possibility by simulating the impedance with a displaced wire. The first resonance is somewhat modified by a radial wire displacement pointing to an asymmetric U-shaped electric monopole field. The impedance at higher frequencies shows a stronger dependence on the wire placement pointing to a dipole component which is coupled to the busbar and depends on the termination. 

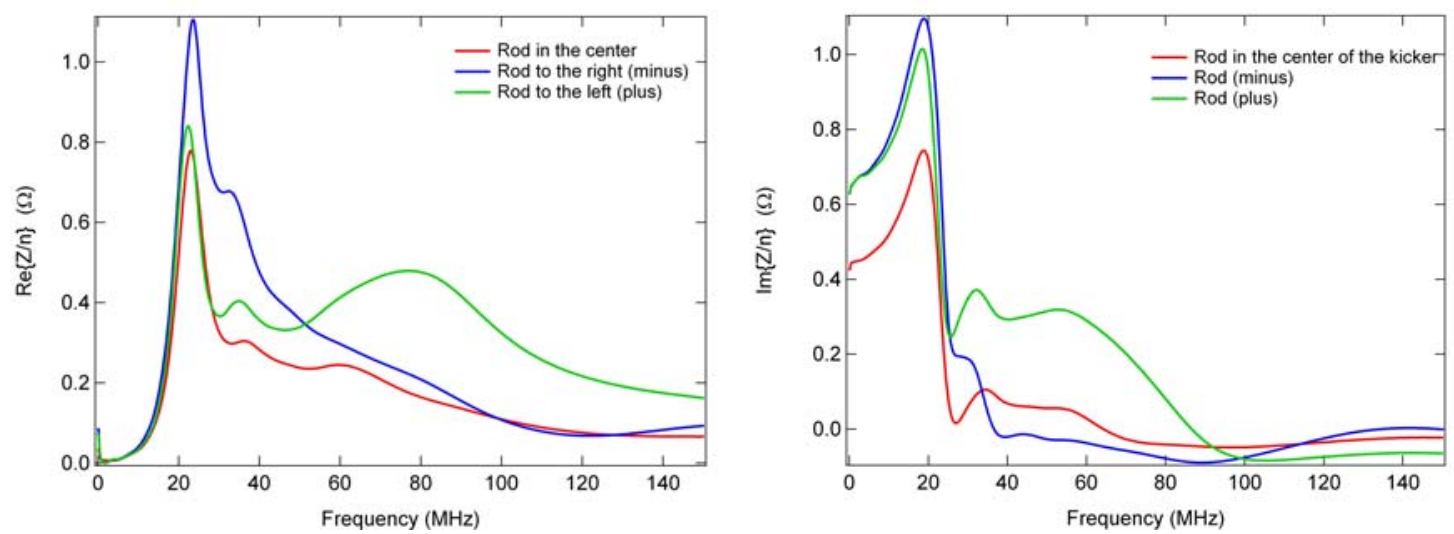

FIGURE 12: Simulation of the kicker impedance with the wire off-centered.

\section{- Discussions and Conclusion}

From the simulation results it is clear that the broad peak around 20 to $40 \mathrm{MHz}$ comes from the ferrite since once we change the ferrite properties (from $\varepsilon_{\mathrm{r}}=10$ to 1 ) we modify the response around this frequency. It is possible also to notice that there is a good

qualitative and quantitative agreement between the simulated data for the ferrite CDM5005 (with $\varepsilon_{\mathrm{r}}=10$ ) and the measurements for this broader peak.

\section{References}

1. RHIC Design Manual, available at http://www.agsrhichome.bnl.gov/NT-share/rhicdm/.

2. H. Hahn et. al., "The RHIC Abort Kicker System”, PAC99, New York, USA 1999, p. 1100 .

3. H. Hahn, Interpretation of coupling impedance bench measurements, Phys. Rev. ST AB, 7, 012001 (2004)

4. A. Ratti, "The Resistively Matched Transition for Measuring the Coupling Impedance of RHIC Devices", EPAC94, London 1994, vol. 2, p. 1262.

5. H. Hahn and A. Ratti, "The Coupling Impedance of the RHIC Injection Kicker System”, AD/RHIC/RD-105, August 1996.

6. H. Hahn and D. Davino, "Transverse Coupling Impedance of the RHIC Abort Kicker”,C-A/AP/52, May 2001.

7. H. Hahn and D. Davino, "RHIC Abort Kicker with Reduced Coupling Impedance", EPAC02, Paris 2002, p.1502. 